\title{
A Bipedal Walking Robot with Efficient and Human-Like Gait*
}

\author{
Steven Hartley Collins \\ Dept. of Mechanical Engineering \\ University of Michigan \\ Ann Arbor, MI 48109 \\ shc@umich.edu
}

\author{
Andy Ruina \\ Theoretical and Applied Mechanics \\ Cornell University, \\ Ithaca, NY 14850 \\ ruina@cornell.edu
}

\begin{abstract}
Here we present the design of a passivedynamics based, fully autonomous, 3-D, bipedal walking robot that uses simple control, consumes little energy, and has human-like morphology and gait. Design aspects covered here include the freely rotating hip joint with angle bisecting mechanism; freely rotating knee joints with latches; direct actuation of the ankles with a spring, release mechanism, and reset motor; wide feet that are shaped to aid lateral stability; and the simple control algorithm. The biomechanics context of this robot is discussed in more detail in [1], and movies of the robot walking are available at Science Online and http://www.tam.cornell.edu/ ruina/powerwalk.html. This robot adds evidence to the idea that passive-dynamic approaches might help design walking robots that are simpler, more efficient and easier to control.
\end{abstract}

Index Terms-efficiency; locomotion; biped; passivedynamic;

\section{INTRODUCTION}

A robot can help test theories of locomotion in ways that are impossible with human subjects; in a robot, one explicitly knows the control strategies being employed and can thus evaluate their qualities. On the other hand, machines that are simple, efficient and easy to control are more likely to be practical as robots. Walking robots based on the mainstream control paradigm of precise jointangle control seem deficient for both purposes by their large energy demands which are not human like and limit autonomous operation times. In contrast, walking robots based on passive-dynamic principles can have human-like efficiencies, and thus more practical energy use requirements [1]. We describe one such powered robot here.

\section{Simulation}

The modeling and analysis of passive-dynamic devices is still essentially in the manner of McGeer [2], and is also well documented in [3]-[6]. In brief, a physical model is conceived, usually as a set of rigid bodies and springs connected by free-swinging joints. In numerical simulation, a discrete nonlinear step-to-step function is generated from the model, a root finding method employed to find fixed points, and local stability evaluated. An approximately

\footnotetext{
${ }^{*}$ This work is supported by two NSF Biomechanics Grants
}

locally optimal set of mass parameters is chosen based on a combination of stability and convenience.

However, direct full simulation was not used as a tool in the development of this robot. The mass distribution and geometry (Fig. 1) are based on a previous fully passive machine which walked down a shallow slope [5]. We were confident that with a passively capable foundation, it would be fairly easy to add a small amount of power without destroying the basic gait cycle. We were reticent to model this design because of the ambiguities involved in modeling its feet. In retrospect, not simulating the device beforehand might have been a mistake; we could instead have redesigned the feet to be of a style more easily modeled, as discussed below.

\section{MechaniCAl Design}

The fully autonomous $12.7 \mathrm{~kg}$ robot walks at $0.44 \mathrm{~m} / \mathrm{s}$ and has two arms, two $0.81 \mathrm{~m}$ long legs, a small torso, and wide, curved feet (Fig. 1). There are 5 internal degrees of freedom: one free hip joint, two periodically locked knees, and two controlled ankle joints. The torso is kept upright with a leg-angle-bisecting mechanism. Each arm carries a battery, and the right arm is rigidly attached to the left leg and vice versa. The robot's mass distribution and geometry are based on [5].

\section{A. Hip and torso design}

At the hip, a small torso and both legs are joined together by a passive 1 degree of freedom joint (Fig. 2). The torso's center of mass is above this joint and an angle bisecting mechanism constrains the torso angle to be the average of the leg angles, described in detail in the caption of figure 2. Such a mechanism has minimal effect on stability [7]. Each leg has a rigid connection through the hip to the contralateral arm. Control electronics are housed in the torso, and the main drive motors are located on each hip, connected to the feet by a cable that runs through the knee.

The torso was added using a hip angle bisecting mechanism so as to maintain a small number of degrees of freedom. However, there is no equivalent mechanical constraint in humans, and the bisecting mechanism results in larger 


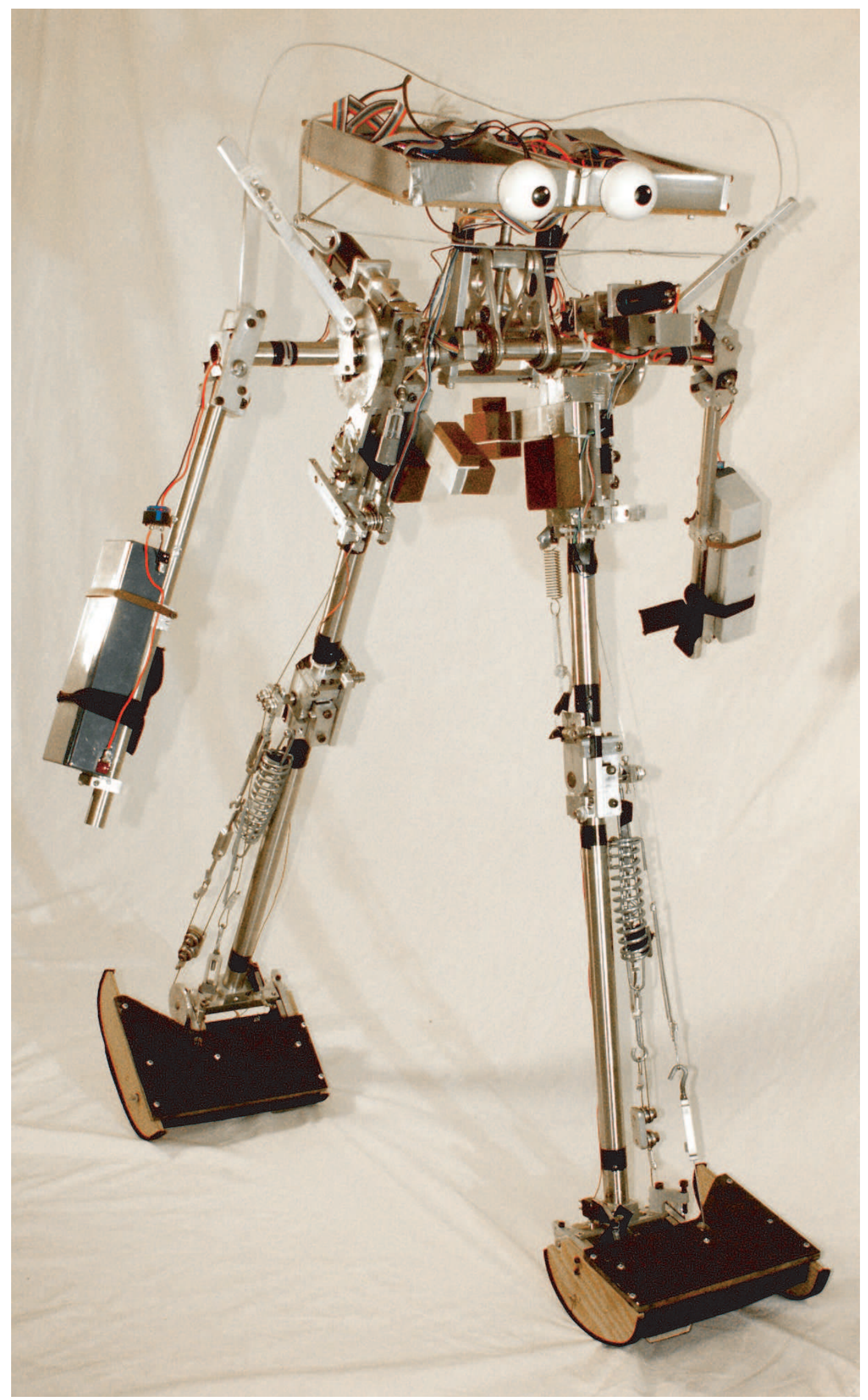

Fig. 1. Bipedal walking robot with efficient and human-like gait. Photo by William Seidel. 
angular displacements of the torso than occur in human gait, due to asymmetry in the swing and stance leg motions.

\section{B. Arm and leg design}

Each thigh is rigidly attached to the contralateral arm, creating one rigid body that acts as the upper leg. These arms are important because they help reduce the tendency of the machine to rotate in yaw (i.e. steer or rotate about a vertical axis). Basically, the arms allow balance of the angular momentum of the system without body rotation [5], [8]. Like the machine in [5], this robot was originally designed with arms that also moved inwards and outwards as they swing. However, we found that this robot was most stable without this in-and-out motion. The vestigial shoulder ab/adductors can be seen in figure 1 . The thigh to shank length and mass ratios are 0.91 to 1 and 3.3 to 1 , respectively, which is important to the passive dynamics of the system.

\section{Knee design}

The thigh and shank shank are joined via a knee joint with a hyperextension limit and locking mechanism, described in detail in (Fig. 3). The knee joint rotates freely when not locked. When the knee reaches full extension midway through swing, so-called "knee-strike", the locking machanism engages, and the knee remains locked in full extension throughout the remainder of swing and during stance. At the beginning of leg swing, a solenoid activates to disengage the knee latch. Thus, the knee motion is largely unactuated. Also at the knee joint is a wire guide that passes the main drive cable through the center of rotation of the knee joint. This allows for power transmission to the ankle joint with minimal effect on knee motion.

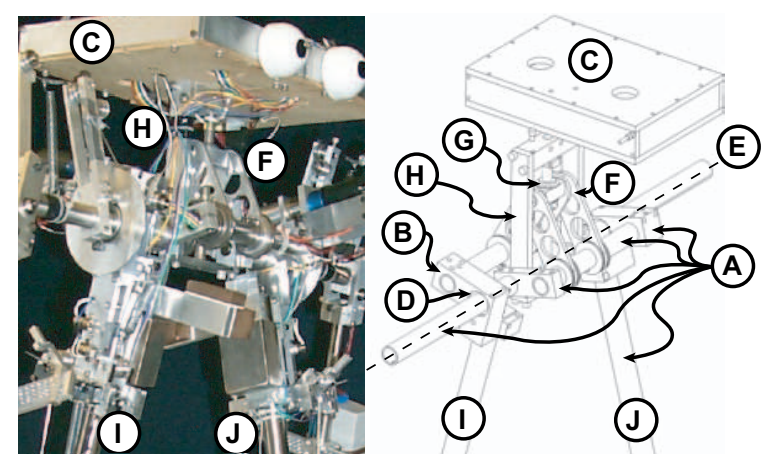

Fig. 2. Photograph (left) and CAD rendering (right) of the hips and torso. The main hip joint consists of three rigid bodies, (A) which is itemized, (B) which is a reflection of (A) through a centered vertical axis, and (C), the torso. (A) and (B) intersect at two hinge joints, (D), resulting in a 1 d.o.f. joint with axis (E). A four-bar linkage consisting of (A), (B), and two sets of $(\mathrm{F})$ moves a slider $(\mathrm{G})$ along a shaft rigidly connected to the torso. This mechanism maintains the absolute angle of the torso (i.e. the angle of $(\mathrm{H})$ ) at the average of the absolute angles of the thighs (I) and (J). Arms attach at the left end of (A) and the right end of (B).
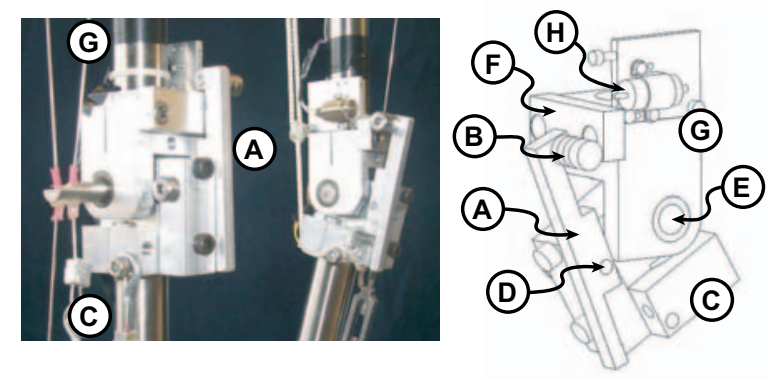

Fig. 3. Photograph (left) and CAD rendering (right) of the device's knees. The photograph shows the right knee locked and left knee in an unlocked configuration. A latch arm (A) with roller (B) is attached to the shank (C) via a hinge at (D). As the knee extends, rotating about the shaft $(\mathrm{E})$, the roller moves along the latch surface $(\mathrm{F})$ which is rigidly attached to the thigh $(\mathrm{G})$. When the roller reaches the horizontal top surface of $(\mathrm{F})$, a spring clicks it into place which locks the knee from flexing until kicked open by solenoid $(\mathrm{H})$. An early version of the wire guide is pictured.

\section{Ankle design}

Redirecting the center of mass velocity at the step-tostep transition (heel strike) is the primary cause of energy loss, and thus energy use, in both passive-dynamics-based walking robots and human walking [9], [10]. In powered walking, adding energy with a "push-off" impulse from the stance leg just before heel strike is four times as effective as restoring energy after the collision has occured [11], [12] because it simultaneously restores energy and reduces the ensuing collision.

Because of the potential energetic benefits and similarity to human walking, power for level-ground ambulation was provided by ankle-extension push-off of the trailing foot. In order to obtain powerful impulses at push-off while minimizing motor power requirements, relatively small motors store energy in springs during leg swing, which are released to perform push-off. This drive mechanism (Fig. 4) also ensures that a known amount of energy is added to the robot with each step, helping the small-perturbation (eigenvalue) stability.

We found that preemptive push-off led to gait instability and greater stresses in mechanical components. We speculate that the gait instability was due to a high sensitivity to the timing of push-off; pushing off a bit too late meant losing four times the energy at heel strike as pushing off right on time. We surrendered possible gains in energy effectiveness in trade for more robust hardware and greater simplicity of control by providing push-off at a force lower than the weight of the device. This choice mechanically constrained push-off to occur directly after heel strike, solving timing issues.

\section{E. Foot design}

Each leg has at its base a foot attached via the actuated ankle joint (Fig. 5). Each foot consists of an inner and outer rail of different curveature, such that the device 
tends to rock side to side as it rolls forward. This allowed the current robot and its predecessor [5] to move from a 2-D concept to 3-D reality with a minimum of design modifications.

However, both machines are highly sensitive to the stiffness and geometry of the foot rails, and would only walk stably for a small range of foot shapes and stiffnesses. This highlights perhaps the greatest shortcoming of this robot: that lateral stability is achieved by keeping the center of pressure in between the widely spaced foot rails. A side effect of the wide, stiff feet is that there is an indeterminacy at heel strike collisions: if one rail strikes just barely before the other, the state just after the collisions is significantly different than if the order were reversed. Additionally, center of pressure can easily reach the rails in some motions, in which case the robot slips and yaws about the point of contact. As mentioned, the wide, curved feet are difficult to model in computer simulation. Greater compliance of the feet would solve these issues, but require active control for lateral balance.

\section{Control Design}

There may be benefits to keeping processing and sensing requirements low in autonomous robots, such as conserving space, energy, and mass, or leaving processor power for thinking about more interesting problems, like deciding where to go. This robot uses a very simple finite-statemachine control algorithm.
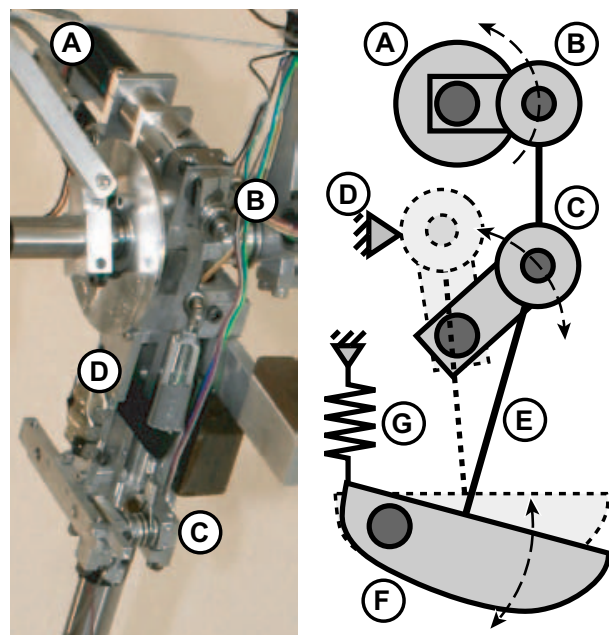

Fig. 4. Photograph (left) and schematic (right) of the drive train. Just after push-off, a DC motor (A), in series with a one-way rotary clutch, drives a motor crank (B). A cable attached to the end of the motor crank via a bearing pulls up an over-center latch (C) until it locks in place against an adjustable stop (D). A cable (E) running from the over-center latch through the knee and to the foot (F) pulls the foot into ready position, stretching a large spring, (G) (visible in Fig. 5). At push-off, a solenoid at (D) moves the over-center latch back past its equilibrium point and the ankle extends, torqued by the spring. The motor crank is pulled along passively as the one-way clutch is rotated in its free direction.

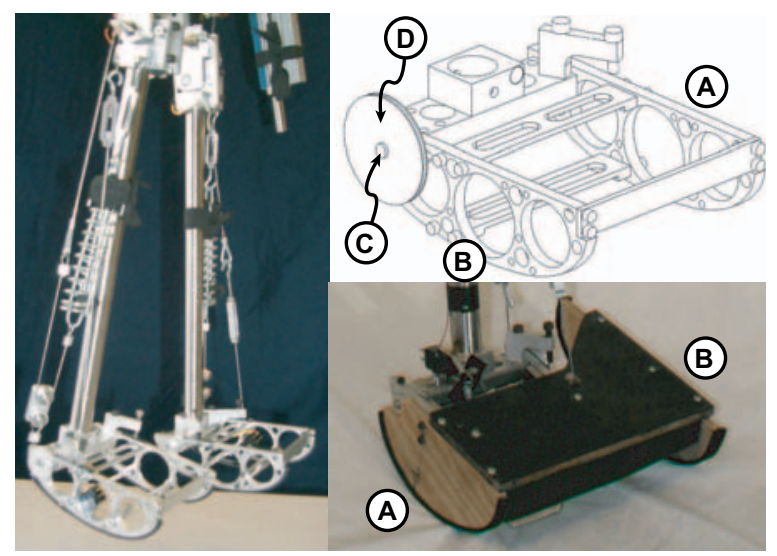

Fig. 5. Photographs (original on left, final on bottom) and CAD rendering (top) of the device's feet. The stiff wide feet consist of rigidly connected inner (A) and outer (B) rails. These rails have different shapes which cause the device to rock back and forth as it rolls forward. The foot rails are pivoted about the ankle joint (C), which is acted upon by a motor and powerful spring through a pulley (D).

\section{A. Controller, sensor, and actuator specifications}

Electronics are located in the torso/head. A state machine with 8 binary inputs and outputs is implemented in 68 lines of $\mathrm{C}++$ code on an Atmel AT90S8515 chip running on an ATSTK500 standard development board. A second board with relays and resistors, (used for signal conditioning) connects the board to actuators and sensors.

The control hierarchy is that of a finite state machine, i.e. a set of simple behaviors that switch between each other, in order, upon appropriate stimulus. In this implementation, all inputs are in the form of switches and all outputs are on-off activations. During the first state, Left Leg Swing, all actuators are unpowered and the left knee latch passively locks at knee strike. When switches below the left foot detect impending heel strike, the state switches to Right Toe-Off. This begins a timed activation of the solenoids that release the plantar-flexor spring of the right foot (Fig. 4). When switches detect full foot extension, the state switches to Right Toe Return. During this state, a 9.5 Watt $6.4 \mathrm{oz}$ MicroMo ${ }^{\circledR}$ motor is activated, slowly retracting the foot and restoring spring energy. A timed activation of the solenoids simultaneously unlocks the right knee. When a switch on the motor indicates full foot retraction, the state switches to Right Leg Swing, and the motor is deactivated. The machine then swaps left and right legs and goes to the initial state. Taking all sensing, including the sensing of internal degrees of freedom which could in principal be made open loop, about 20 bits of information per step flows to the processor. Environmental sensing, i.e. the instant of foot contact, is about one third of that. 


\section{Results}

\section{A. Gross walking kinematics}

This robot was designed to perform one task: walk forwards at constant speed. It first walked steadily on July 31, 2003. In its most robust configuration, the $12.7 \mathrm{~kg}$ robot walked at $0.44 \mathrm{~m} / \mathrm{s}$ with an average step period of 0.85 seconds. Because push-off occurs after heel strike, there was a double support phase, during which both feet were in contact with the ground, lasting about 0.11 seconds. We believe that the gait of this machine is rather human-like in appearance (movies at http://www.tam.cornell.edu/ ruina/powerwalk.html).

\section{B. Energetic Consumption}

A key issue in locomotion is energy use [13]. The energy efficiency of level locomotion is usefully measured by the specific cost of transport $c_{e t}=$ (energy used)/(weight)(distance traveled) [1], [14]. A related measure is mechanical energy efficiency $c_{m t}$ which assigns an energy cost only to the mechanical work of the actuators.

Energy measurements were taken during walking trials using an off-board digital oscilloscope connected to the robot with fine wires. At $500 \mathrm{~Hz}$, the oscilloscope measured battery voltage on one channel and the voltage drop across a $1 \mathrm{ohm}$ power resistor in series with the batteries on another (Fig. 6). The average power consumption was 10.9 Watts. Mechanical energy use was measured in experimentally simulated push-off trials; the force at each foot contact point was measured as the ankle moved through its extension range, and this force was integrated to find mechanical work per step. Given the step period, we calculated an average mechanical power of 2.9 Watts. Given the weight and walking speed of the robot, these electrical and mechanical power consumptions yield $c_{e t} \approx 0.20$ and $c_{m t} \approx 0.055$.

Table I lists $c_{e t}$ and $c_{m t}$ for several locomotive devices including several walking robots, our robot, and human beings. Asimo, representative of joint-angle-controlled robots, uses electric gear motors, which can have conversion efficiencies (electric energy to mechanical work) higher than $70 \%$. However, Asimo's $c_{e t}$ is an order of magnitude greater than a human's, indicating that much more mechanical energy is being produced, and absorbed, by its actuators than in human gait. The Spring Flamingo allows natural dynamics to contribute to its walking motion, reducing the mechanical work done on its limbs (and its $c_{m t}$ ) [15]. However, the Series Elastic Actuators used for force control produce and absorb mechanical work internally, so the $c_{e t}$ remains high. T.U. Delft's Denise harnesses passive dynamics and uses pneumatic McKibben muscle actuators with high conversion efficiency. However, the onboard $\mathrm{CO} 2$ storage system requires an inefficient down-regulation of pressure, so the $c_{e t}$ remains high. Finally, our robot utilizes passive dynamics to keep $c_{m t}$ low, and a zero-negativework actuation scheme implemented with electric motors to keep $c_{e t}$ low as well. All three robots that utilize natural dynamics have $c_{m t}$ similar to that of McGeer's unpowered walking machine. Likewise, our robot has $c_{e t}$ and $c_{m t}$ similar to humans.

As an analogy, airplanes and gliders have become more efficient than the Wright Flyer over the past century, yet modern helicopters still consume an order of magnitude more energy. This discrepancy is not a matter of available technology but rather a direct result of mechanical design and control strategy.

\section{Robustness}

This robot is a proof-of-concept prototype, not a production machine, and it was developed with $\$ 10 \mathrm{~K}$. As is typical for this type of robot, it did not stand up well to long periods of testing, with wires and bearings breaking frequently. When the Cornell robot was best tuned it would walk successfully at about $30 \%$ of attempts. Failed launches were due to inadequate matching of proper initial conditions, most often ending with foot scuff of the swing leg. The robot is mildly unstable in heading, so once it was launched, the primary failure mode was walking off of the (narrow) walking table or walking into a wall. Because it walked 10 or more steps many times, the gait is clearly stable (although not very) for both lateral and sagittal balance.

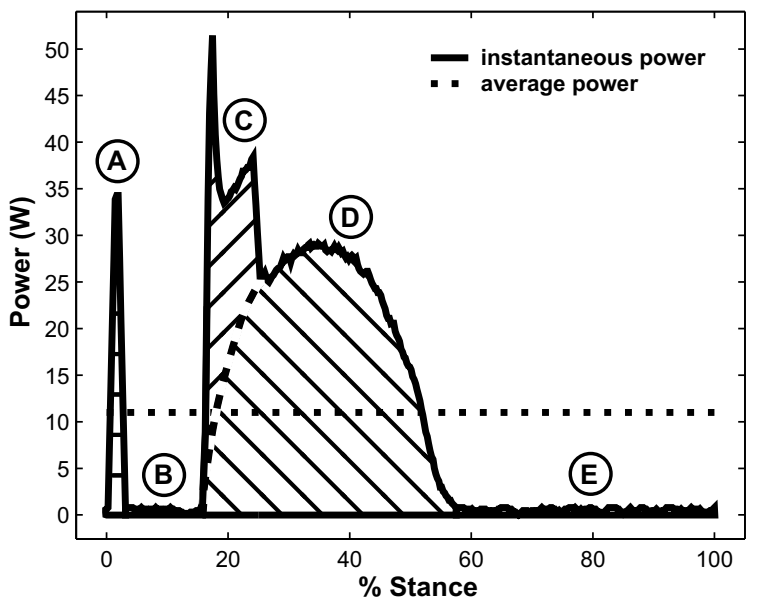

Fig. 6. Total electrical power used over one step. Area under this plot is equivalent to (scaled) energy. Solenoids use significant energy to trigger toe-off (A) and unlatch the swing knee (C). A DC motor uses approximately $70 \%$ of the energy in returning the ankle spring to its stretched position (D), which is the electric contribution towards propulsive energy. During ankle extension (B) and late leg swing (E) there is no actuation, and control electronics use relatively little power. Mechanical work is done during (B). 
TABLE I

ESTIMATED SPECIFIC COST OF TRANSPORT, $c_{e t}$, AND MECHANICAL ENERGY EFFICIENCY, $c_{m}$, OF SEVERAL LOCOMOTIVE DEVICES*

\begin{tabular}{llll}
\hline \hline & & $c_{e t}$ & $c_{m t}$ \\
\hline \hline Walking & Honda's Asimo $^{\alpha}$ & 3.2 & 1.6 \\
Robots: & T.U. Delft's Denise $^{\beta}$ & 5.3 & 0.08 \\
& MIT's Spring Flamingo $^{\gamma}$ & 2.8 & 0.07 \\
& Our Robot & 0.20 & 0.055 \\
& McGeer's Dynamite $^{\delta}$ & - & 0.04 \\
\hline Humans $^{\epsilon}:$ & Walking $^{\zeta}$ & 0.2 & 0.05 \\
\hline Flying & Modern Helicopter $^{\eta}$ & 1.6 & 0.4 \\
Machines: & Wright Flyer & 0.72 & 0.18 \\
& Boeing 747 $^{\iota}$ & 0.12 & 0.05 \\
& Modern Glider $^{\kappa}$ & - & 0.02 \\
\hline Other $^{\lambda}:$ & Efficient Auto & 0.06 & 0.015 \\
& Cyclist & 0.04 & 0.01 \\
& Freight Train & 0.012 & 0.003 \\
& Freighter & 0.004 & 0.001 \\
\hline \hline
\end{tabular}

* Values represent best performance at a constant speed and average weight. $\alpha$ Asimo $c_{e t}$ from publicly available data $(38.4 \mathrm{~V} 10$ amp-hour battery used in 30 minutes, weight of $510 \mathrm{~N}$, walking speed of $1.6 \mathrm{~km} / \mathrm{hr}$ ), $c_{m t}$ assumes $50 \%$ drive train efficiency. $\beta$ Denise values from [1]. $\gamma 2-\mathrm{D}$, not autonomous. Spring Flamingo values from [15]. $\delta$ Dynamite values from [2]. $\epsilon$ Measured by net VO2 (see [16], [17]). $\zeta$ Human values from [9]. $\eta$ Helicopter values based on: $1500 \mathrm{~kg}, 110$ gal. jet fuel, $600 \mathrm{~km}$ trip. $\theta$ See [18]. $\iota$ Boeing 747 values based on: $350,000 \mathrm{~kg}, 5$ gal. jet fuel per mile. $\kappa$ Glider values based on a glide ratio (distance traveled per distance fallen) of 50. $\lambda$ Other values from [14].

\section{CONCLUSiOnS}

We have presented a design for an autonomous, 3-D bipedal walking robot with efficient and human-like motions. This project might serve as another example of a general strategy for minimizing energy use in walking robots: careful consideration of the natural dynamics of the mechanical system, control design that utilizes these dynamics, and efficient actuation which produces, but rarely absorbs, mechanical work. We believe that this passive-dynamic design method will help to build walking robots that are simpler, more efficient, easier to control, and therefore more practical. For an in depth consideration of the broader implications of our results, please see [1].

\section{FUTURE WORK}

The next generation of passive-dynamics-based robots should solve the following design challenges, motivated above: improved actuation systems that exhibit both high control authority and low impedence with high conversion efficiency; lateral balance through controlled placement of compliant, anthropomorphically-sized feet; compliant load acceptance (heel strike) and optimal push-off timing through double stance; increased robustness through regulation of push-off energy; torso control; and arm control. Implications for the design of advanced foot prostheses are also suggested by our results.

\section{ACKNOWLEDGMENT}

Stephen Keast, Mario Gomes, Andrew Dressel, and Michael Sherback helped with design and construction.
David Cabrera helped with testing. Martijn Wisse and Art Kuo collaborated on plans for future work.

\section{REFERENCES}

[1] S. H. Collins, A. Ruina, M. Wisse, and R. Tedrake, "Efficient bipedal robots based on passive-dynamic walkers," Science, 2005, in press.

[2] T. McGeer, "Passive dynamic walking," Int. J. Robotics Res., vol. 9, pp. 62-82, 1990.

[3] M. Coleman and A. Ruina, "An uncontrolled toy that can walk but cannot stand still," Phys. Rev. Lett., vol. 80, no. 16, pp. 3658-3661, 1998.

[4] M. S. Garcia, "Stability, scaling, and chaos in passive dynamic gait models," Ph.D. dissertation, Cornell University, Theoretical and Applied Mechanics, may 1996.

[5] S. H. Collins, M. Wisse, and A. Ruina, "A three-dimensional passive-dynamic walking robot with two legs and knees," Int. J. Robotics Res., vol. 20, no. 7, pp. 607-615, 2001.

[6] M. Wisse and J. van Frankenhuyzen, "Design and construction of mike; a 2d autonomous biped based on passive dynamic walking," in Proc. Conf. Adaptive Motion of Animals and Machines. Kyoto, Japan: AMAM, 2003.

[7] M. Wisse, "Essentials of dynamic walking: Fill me in," Ph.D dissertation, T.U. Delft, Man Machine Systems, September 2004

[8] H. Elftman, "The function of arms in walking," Human Biology, vol. 11 , pp. 529-535, 1939.

[9] J. M. Donelan, R. Kram, and A. D. Kuo, "Mechanical work for step-to-step transitions is a major determinant of the metabolic cost of human walking," J. Exp. Biol., vol. 205, pp. 3717-3727, 2002.

[10] J. E. A. Bertram and A. Ruina, "Multiple walking speed-frequency relations are predicted by constrained optimization," J. Theor. Biol., vol. 209, no. 4, pp. 445-453, 2001.

[11] A. D. Kuo, "Energetics of actively powered locomotion using the simplest walking model," J. Biomech. Eng., vol. 124, pp. 113-120, 2002.

[12] A. Ruina, J. Bertram, and M. Srinivasan, "A collisional point-mass model for the energetic cost of the down-to-up transition in legged locomotion: running, galloping and walking," Journal of Theoretical Biology, 2005, in press.

[13] R. M. Alexander, Principles of Animal Locomotion. Princeton, NJ: Princeton Univ. Press, 2003.

[14] V. Radhakrishnan, "Locomotion: Dealing with friction," Proc. Natl. Acad. Sci. USA, vol. 95, pp. 5448-5455, 1998.

[15] J. Pratt, "Exploiting inherent robustness and natural dynamics in the control of bipedal walking robots," Ph.D. dissertation, MIT, 2000.

[16] E. Atzler and R. Herbst, "Arbeitsphysiologische studien," Pflg. Arch. ges. Physiol., vol. 215, pp. 291-328, 1927.

[17] N. H. Molen, R. H. Rozendal, and W. Boon, "Graphic representation of the relationship between oxygen-consumption and the characteristics of normal gait of the human male," Proc. Kon. Ned. Akad, vol. 75, pp. 305-314, 1972.

[18] "Flight and flying," in Encyclopaedia Brittannica, 11st ed. Encyclopaedia Britannica, Inc, 1910, vol. 10, p. 514 